
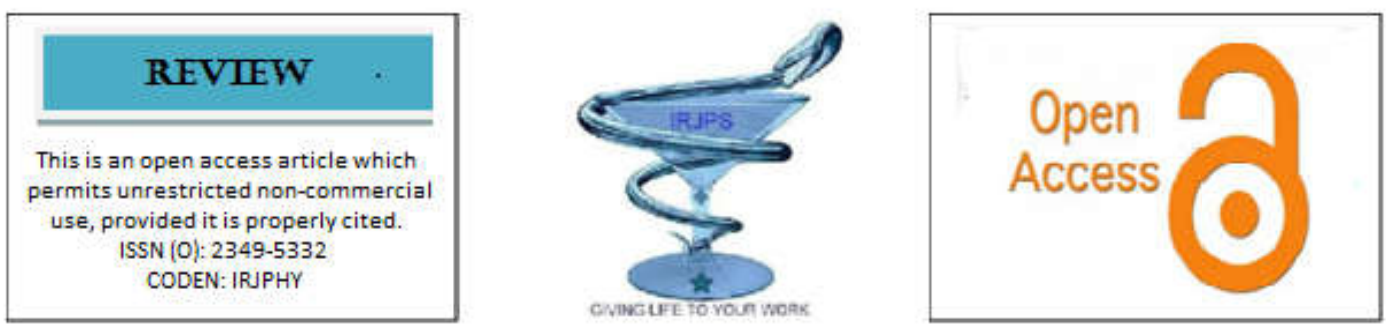

\title{
REVIEW ON ORIGIN, GENOMIC STRUCTURE AND REPLICATION OF CORONA VIRUS \& COVID - 19
}

\author{
Swati Sharma ${ }^{1}$, Preeti Chauhan ${ }^{2}$, Deepika Sharma ${ }^{3 *}$ \\ Department of Pharmaceutical Chemistry, Abhilashi College of Pharmacy, Nerchowk, Mandi \\ Department of Pharmacognosy, Abhilashi College of Pharmacy, Nerchowk, Mandi \\ *Department of Pharmacology, Abhilashi College of Pharmacy, Nerchowk, Mandi
}

Submitted on: 18.06.2020; $\quad$ Revised on: 26.06.2020; $\quad$ Accepted on: 05.07.2020

\begin{abstract}
:-
Corona virus is derived from latin word corona which means "crown" and having appearance like solar corona, due to this the surface is covered with club shaped protein spikes. These are the viruses which infect humans and large varieties of animals which includes both birds and mammals. SARS-CoV (Severe Acute Respiratory Syndrome) identified in southern China in 2003, and MERS-CoV (Middle East Respiratory Syndrome), identified in Saudi Arabia in 2012. They are positive sense single-stranded RNA genomic viruses. Corona viruses have club-shaped $\mathrm{S}$ proteins on the envelope, which bind to the cells and undergo membrane fusion to gain entry into the cytoplasm. The cleavage of S proteins varies among Corona viruses. Covid-19 is caused by infection of severe acute Corona virus (SARS-CoV-2). As proposed by the International Committee for Taxonomy of Viruses, CoVs are further categorized into four main genera:- Alpha, Beta, Gamma and DeltaCorona viruses based on sequence comparisons of entire viral genomes. HCoVs are identified to be either in the Alpha or Beta Corona virus genera which includes AlphaCorona viruses (HCoV-229E and HCoV-NL63) and BetaCorona viruses (HCoV-HKU1, SARS-CoV, MERS-CoV and HCoV-OC43).
\end{abstract}

KEYWORDS:-Corona virus, Positive-sense RNA viruses, SARS-CoV, MERS-CoV

Corresponding Author: Deepika Sharma

Phone: +91-9418024895

Email: deepikasharma.bhushan@gmail.com
Indian Research Journal of Pharmacy and Science; 25(2020)2299-2305; Journal Home Page: https://www.irjps.in DOI: 10.21276/irjps.2020.7.2.20 


\section{INTRODUCTION:-}

Corona viruses are the viruses which infect humans and large varieties of animals which includesboth birds and mammals. Two different syndrome have evolved SARS-CoV (Severe Acute Respiratory Syndrome) identified in southern China in 2003, and MERS-CoV (Middle East Respiratory Syndrome), identified in Saudi Arabia in 2012. $[1,2]$ Corona viruses comes under family Coronaviridae, subfamily orthocoronavirinae, and order Nidovirales. They are positive sense singlestranded RNA genomic viruses. Corona virus is derived from latin word corona which means "crown" and having appearance like solar corona, due to this the surface is covered with club shaped protein spikes. Corona viruses are well-established pathogens of humans and animals while the toroviruses are recognized as causes of animal diarrhoea.[3]Novel Corona virus (COVID-19) was first reported from Wuhan, China in December 2019. Then the disease was rapidly spread to different regions of China and also to the neighbouring countries like Japan, India, Singapore, Thialand, and South-Korea, Malaysia and to the western countries. In the mid of February the total number of cases reported were 43,112 which were far exceeded to the number of patient with SARS. Corona viruses cause acute and chronic respiratory, enteric and central nervous system (CNS) diseases in many species of animals, including humans. The Corona virus is the virus responsible for causing what is known as the Middle East Respiratory Syndrome. This virus causes severe viral infections with high mortality rates.[4]

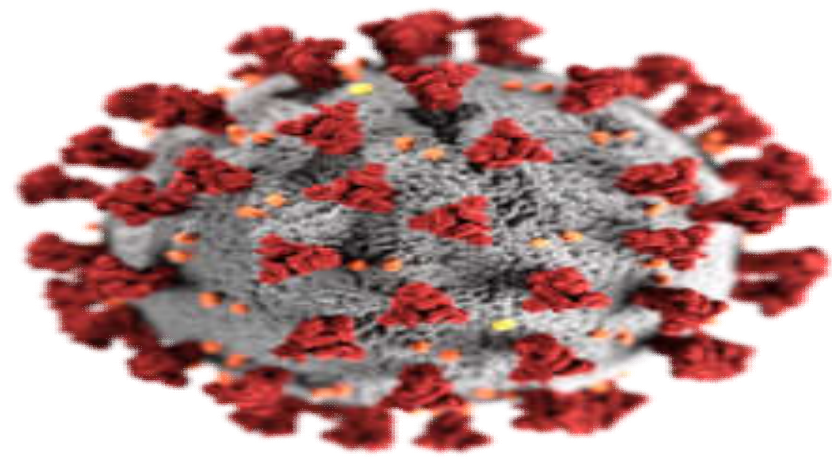

Fig 1: Structure of Corona virus

Taxonomy of Corona virus

Family: Coronaviridae

Order: $\quad$ Nidovirales

Subfamily: Orthocoronavirinae

\section{GENOMIC STRUCTURE AND} MORPHOLOGY OF CORONA VIRUS:-

All Corona viruses consists of glycoprotein membrane,(M) and large spike glycoprotein,(S).
Members of betacoronovirus have shorter spikelike protein, hemagglutinin esterase (HE). These type of viruses includes:- Human Corona virus OC43 (HCV-OC43) and bovine Corona virus (BCV).The outer surface of the lipid bilayer contain small portion of the amino terminus.[5] The glycoprotein which makes up the large spike, S, has an apparent molecular weight is 150 to $200 \mathrm{~K}$ and appears to be a dimer held together by non-covalent bonds. Nucleocaspid protein $\mathrm{N}$ is present in helical 
nucleocaspid and the plusstrand. The genomic RNA is enclosed in viral envelop.Cysteine residue is located on the lower part of lipid bilayer and act as terminal domain. At the time of maturation and intacellulartansport some molecules of $\mathrm{S}$ are breaked up by host cell proteases. All the process occurs in Golgi apparatus and after cleavage two large subunits are formed:- S1 and S2. Hence the cleavage is totally host-cell dependent.Thecarboxyterminal subunit of S2is covalently linked to Palmitic acidand N-linked oligosaccharides are found on both of the subunits of this glycoprotein. Atalkaline pH.aggregation may occur.[6,12,15]

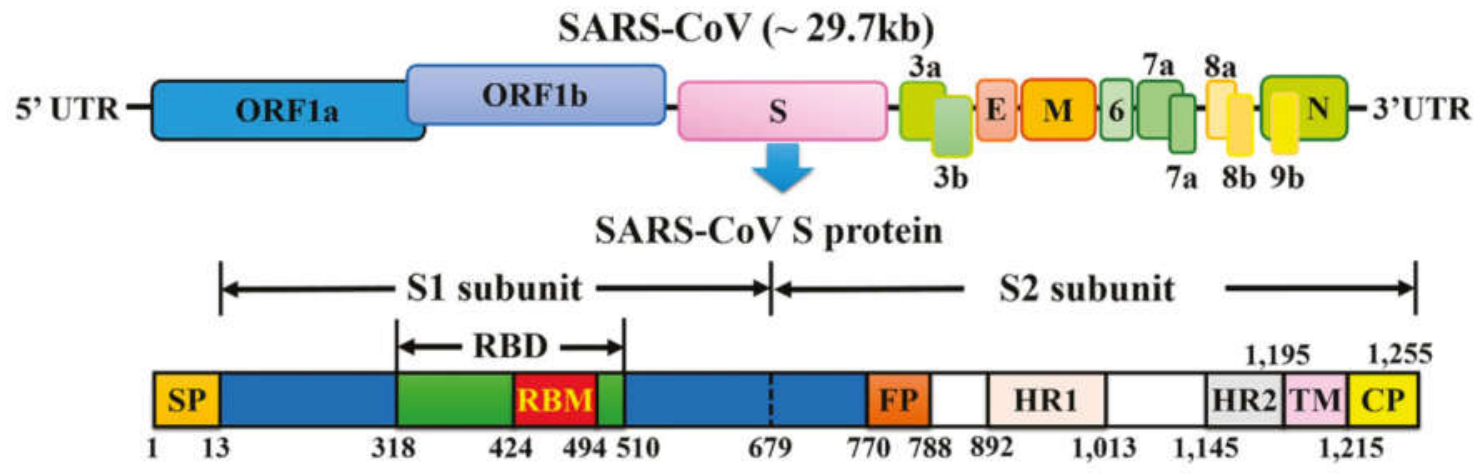

MERS-CoV ( 30.1 kb)

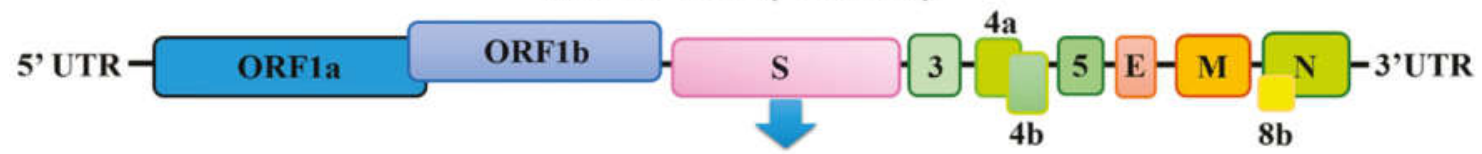

MERS-CoV S protein

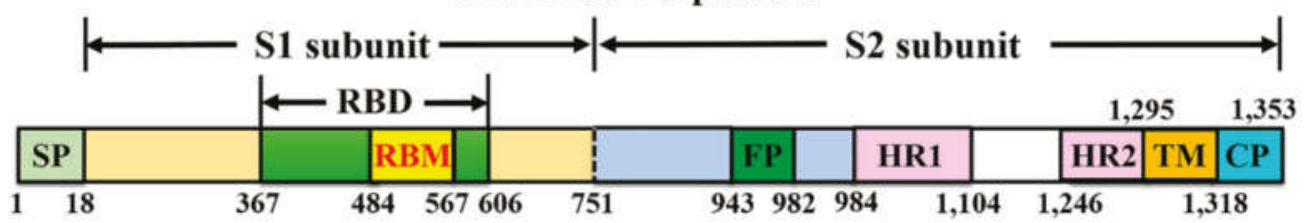

Fig 2: Schematic Representation of the Genome Organization and Functional Domains of S- Protein for SARS-COV and MERS-COV.

\section{CORONA VIRUS REPLICATION CYCLE:-}

After attachment of virus to the host cell, the protease of the host cell cleaves and activates spike protein. The entry of virus occurs through endocytosis or by direct fusion to the host membrane. In host cell virus get uncoated and its genome get enters in the cytoplasm of cell. 5'methylated cap and 3' polyadenylate tail of Corona virus having RNA genome allows RNA to attach to the ribosomes of hostcell for translation process.[7]
Initially the overlapping of virus genome occurs to the host cell to form long chain polyprotein. The proteases enzymes are located inpolyproteinchain which helps in the cleavage of polyprotein into large numbers of multiple non-structural proteins.

Then these non-structural proteins fuse to form a multi-protein replicase transcriptase complex (RTC).This process is RNA-dependent RNA polymerase $(\mathrm{RdRp})$ and involved in the replication and transcription of RNA from RNA strand.Exoribonucleases in non-structural protein provides proofreading function for replication 
process. One of the main functions of the complex is to replicate the viral genome.

RdRp directly mediates the synthesis of negativesense genomic RNA from the positive-sense genomic RNA and this process is followed by the replication of positive-sense genomic RNA from the negative-sense genomic RNA. The other important function of the complex is to transcribe the viral genome.[8]

The synthesis of negative-sense sub genomic RNA is mediated by RdRpfrom positive-sense genomic RNA. This process is followed by transcription of these negative-sense sub genomic RNA to corresponding positive-sense mRNA. [9]

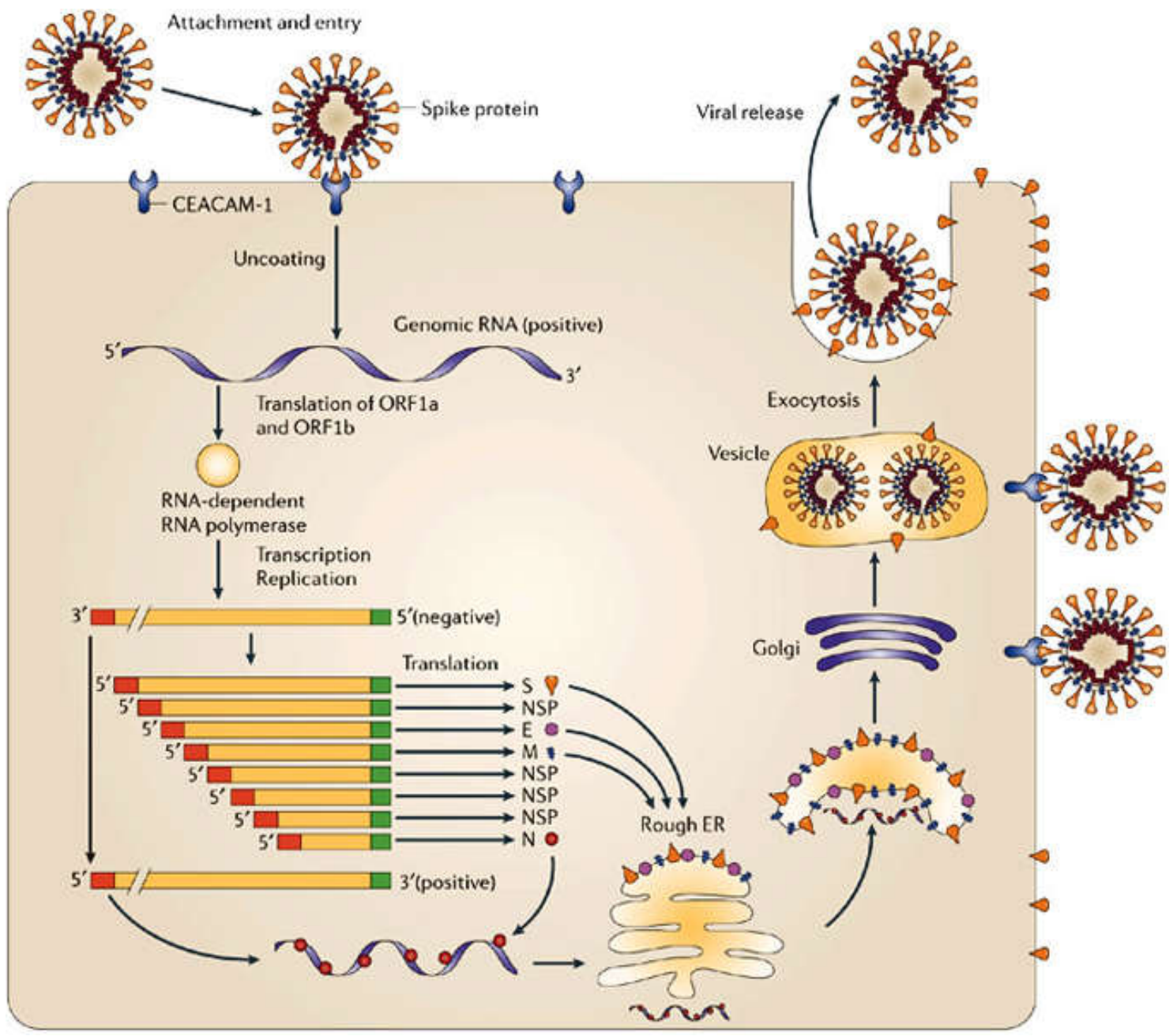

Fig 3: Diagramatically representation of Replication of Corona-virus MHV (Mouse Hepatitis virus)

\section{HUMAN CORONA VIRUS:-}

In SARS-CoV, Corona viruses causes self-limiting respiratory infections in human. Two of these viruses are $\alpha$-Corona viruses $(\mathrm{HCoV}-229 \mathrm{E}$ and HCoV-NL63) while the other two are $\beta$-Corona viruses (HCoV-OC43 and $\mathrm{HCoV}-\mathrm{HKU} 1)$. These viruses cause several diseases like fever, sore throat, pneumonia and upper and lower respiratory tract infection. They cause more severe disease in neonates, elderly, and in individuals with underlying illnesses, with a greater incidence of lower respiratory tract infection in these populations. 
HCoV-NL63 can cause acute laryngotracheitis. $\mathrm{HCoV}-229 \mathrm{E}$ infects mice while $\mathrm{HCoV}-\mathrm{OC} 43$ is related to bovine Corona virus, $\mathrm{BCoV}$. They are capable of infecting mice and several ruminant species. [10]

SARS-CoV possess typical structure having spike protein, nucleoprotein and membrane protein. The spike protein contains 3D-structure in RBD region and maintain vanderwaal forces and these spike portion get attached to ACE2 receptors in humans.[11]

\section{SPREAD OF COVID-19:-}

Wuhan in China becomes the main center of an outbreak of covid in December 2019. On $7^{\text {th }}$ January, a Chinese scientist isolates novel Corona virus from an infected person in Wuhan. This strain of Corona virus known as the severe acute respiratory syndrome Corona virus 2 (SARSCoV2 ), and also referred to as 2019-nCoV. The infection caused by this virus is known as Corona virus disease-2019 (COVID-19). [12]

According to the World Health Organization (WHO), by the middle of February 2020, cases of COVID-19 has been reported in the Western Pacific region (Singapore, Japan, Republic of Korea, Malaysia, Viet Nam, Australia, Philippines, Cambodia), South-East Asia (Thailand, India, Nepal, Sri Lanka), North America (the USA and Canada), Europe (Russian Federation, Spain, Belgium, Finland, Sweden, Germany, France, UK and Italy) and the Eastern Mediterranean region (United Arab Emirates).[13,14]

The transmission of COVID-19 in Wuhan, suggesting that these early infection were due to animal-to-person transmission. However, the later cases or studied showed the indication of humanto-human transmission. The latest guidelines from Chinese health authorities describe three main routes for the transmission of COVID-19:- Droplet transmission, Contact transmission and Aerosol transmission.[15]

Modes of Transmission of Infection:-

1) Direct contact with the droplets dispersed when a patient coughs or sneezes.

2) Indirect contact by touching surfaces or objects contaminated with the virus and then touching the nose, eyes or mouth.

3) Direct contact with infected people, animals and their products.

Droplet transmission occurs when an infected person coughs or sneezes, which are ingested or inhaled by individual nearby in close proximity.Contact transmission occur when a person touches a surface or object contaminated with the virus and subsequently touch their mouth, nose, or eyes and aerosol transmission may occur when respiratory droplets mix into the air, forming aerosols and may cause infection when inhaled high dose of aerosols into the lungs.[16,17]

Control of transmission of COVID-19:Handwashing and social-distancing are the main control for viral transmission. Use of masks, gloves is also recommended. Transmission can also occurs through ocular surface so eye protectors can also be used.[18]

\section{VACCINES AND TREATMENTS:-}

Presently there is no vaccine or specific anti-viral drug available for the treatment of infected person. WHO estimates that near about after 18 months vaccines for COVID-19 to be available. So, presently the provision of treatment is antiinflammatory and anti-viral drugs.

For complicated patients continuous renal replacement therapy (CRRT), invasive mechanical 
ventilation and extracorporeal membrane oxygenation (ECMO) are used as supportive treatment. No specific antiviral drugs have been confirmed effective.

In USA the first patient reported with $\mathrm{CoV}-19$ was treated with remdesivir and others have used antiretrovirals like ritonavir, with trials of both in progress. Recently, Baricitinib has been suggested as a potential drug for the treatment that it might reduce the process of both virus invasion and inflammation. [19,20]

An antiviral drug Remdesivir which is effective against RNA viruses (including SARS/MERSCoV5) infection in mice, cultured cell and nonhuman primate (NHOP) models.

Chloroquine which is widely used as anti-malarial drug is used as potential broadspectrum antiviral drug and block viral infection by increasing endosomal $\mathrm{pH}$ virus/ cell fusion, as well as interfering with the glycosylation of cellular receptors of SARS-CoV. [21]

\section{REFERENCES:-}

1. Susanna KP Laul, Jasper FW Chan. Corona viruses: emerging and re-emerging pathogens in humans and animals. Virology Journal 2015;12:209: 1-3.

2. Katelyn Gostic, Ana CR Gomez, Riley O Mummah,et al. Estimated effectiveness of symptom and risk screening to prevent the spread of COVID-19. Epidemiology and Global Health 2020; 9:e55570: 1-18.

3. YunjeongKima,

SivakoteswaraRaoMandadapub, William

C. Groutasb,et al. Potent Inhibition of Feline Corona viruses with Peptidyl Compounds Targeting Corona virus 3C- like Protease.Antiviral Res 2013

February;97(2): 161-168.

4. BalasubramanianArul,

RamalingamKothai.2019NovelCorona virus:

AmysteriousthreatfromWuhan,China-A currentreview. Int. J.Res. Pharm. Sci.2020;11(SPL)(1): 7-15.

5. David T Brown K, Cavanagh David. Advances in experimental medicine and biology. Corona viruses and their diseases, Vol.276.

6. Yvonne Xinyi Lim, Yan Ling Ng, James P. Tam and Ding Xiang Liu. Human Corona viruses: A Review of Virus-Host Interactions. Diseases 2016; 4, 26: 1-28.

7. Yan-RongGuo, Qing-Dong Cao, Zhong-Si Hong et al.The origin, transmission and clinical therapies on Corona virus disease 2019 (COVID-19) outbreak - an update on the statusMilitary Medical Research $2020 ; 7: 11: 1-10$

8. JustynaMiłek, KatarzynaBlicharzDomańska.Corona viruses in avian species - review with focus on epidemiology and diagnosis in wild birds. J Vet Res 62, $2018 ; 249-255$

9. Anthony R. Fehr, Stanley Perlman, Corona viruses: An Overview of Their Replication and Pathogenesis. Methods MolBiol 2015; 1282: 1-23.

10. Victor M Corman, OlfertLandt, Marco Kaiser, et al. Detection of 2019 novel Corona virus (2019-nCoV) by real-time RT-PCR.Euro Surveill, 25(3)2020.

11. Muhammad Adnan Shereen, Khan Suliman, KazmiAbeer, et al. COVID-19 infection: Origin, transmission and characteristics of human Corona viruses. 
Journal of Advance Research 2020; 24: 91-98.

12. Khan Zakir, Muhammad Khayal, Ahmed Ali,et al. Corona virus outbreaks: prevention and management recommendations.Drugs \& Therapy Perspectives 2020.

13. Hyungjin Kim.Outbreak of novel Corona virus (COVID-19): What is the role of radiologists.European Radiology 2020.

14. LUO Hui, TANG Qiao-ling, SHANG Yaxi, et al.Can Chinese Medicine Be Used for Prevention of Corona virus Disease 2019 (COVID-19)? A Review of Historical Classics, Research Evidence and Current Prevention Programs.Chin J Integr Med.

15. SasmitaPoudelAdhikari, ShaMeng, Yu-Ju $\mathrm{Wu}$, et al.Epidemiology, causes, clinical manifestation and diagnosis, prevention and control of Corona virus disease (COVID-19) during the early outbreak period: a scoping review.Infectious Diseases of Poverty 2020; 9:29.

16. Wei-jie Guan, Rong-chang Chen, Nan-
shanZhong.Strategies for the prevention and management of Corona virus disease 2019.EurRespir J 2020.

17. Lake A Mary. What we know so far: COVID-19 current clinical knowledge and research, Clinical Medicine 2020 Vol 20, No 2: 124-7.

18. Fang Jiang, Liehua Deng, Liangqing Zhang, et al.Review of the Clinical Characteristics of Corona virus Disease 2019 (COVID-19).J Gen Intern Med, 2020.

19. Yaseen M. Arabi1,SrinivasMurth, Steve Webb, COVID-19: a novel Corona virus and a novel challenge for critical care.Intensive Care Med.

20. Anne Catherine Cunningham, HuiPohGoh, David Koh.Treatment of COVID-19: old tricks for new challenges. Critical Care 2020; 24:91.

21. Remdesivir and chloroquine effectively inhibit the recently emerged novel Corona virus(2019-nCoV) in vitro. Cell Research 2020;30:269-271. 